\title{
Phase Behavior of Sphere-Forming Triblock Copolymers in Films
}

\author{
Hong-Ge Tan ${ }^{1 *}$, Qing-Gong Song ${ }^{1}$, Xin-Huan Yang ${ }^{2}$, Ya-Jing Deng ${ }^{1}$ \\ ${ }^{1}$ College of Science, Civil Aviation University of China, Tianjin, China \\ ${ }^{2}$ College of Science, Hebei University of Technology, Tianjin, China \\ Email: *thg75@sina.com.cn
}

Received July 8, 2012; revised August 5, 2012; accepted August 16, 2012

\begin{abstract}
The self-assembly of sphere-forming triblock copolymers confined between two thin homogeneous surfaces is investigated based on mean-field dynamic density functional theory. The morphologies deviating from the bulk sphere-forming phase are revealed, including cylinders oriented perpendicular to the surface, cylinders oriented parallel to the surface, perforated lamellae and lamellae by varying film thickness and surface field strength. The phase diagram of surface reconstruction is also constructed. By comparing the present phase diagram with the other relevant phase diagram for the cylinder-forming triblock copolymer film, the difference between the sphere-forming and the cylinder-forming triblock copolymer thin film is discussed.
\end{abstract}

Keywords: Triblock Copolymer; Mean-Field Dynamic Density Functional Theory; Thin Film

\section{Introduction}

Block copolymer molecules are composed of two or more chemically distinct blocks of monomer units. Since the blocks are joined covalently, they can form microscopic phase separation and are thus forced to form rich ordered microstructures. Those ordered microstructures could be useful in nanotechnology application. For bulk block copolymers, these microdomains have been obtained by controlling the molecules weight, molecules architecture and interaction parameter between different segments (blocks). When a block copolymer melt is confined in the film, two additional factors have to be considered. One is the surface energy, the other is the film thickness. In order to obtain controllable patterns on a nanometer scale, films of block copolymers have received considerable attention. The self-assembly of diblock copolymers confined in films has been studied extensively both theoretically and experimentally [1-4]. Under confinement, diblock copolymers can not only change the orientation, but also alternate between different morphologies deviating from the bulk structure.

In contrast to confined diblock copolymers, phase behavior of confined triblock copolymers is more complicated [5-9]. Stcoker et al. studied surface reconstruction of the lamellar morphology in triblock copolymers by the experiment [5]. Rehse et al. studied the microstructure near the surface in lamellar $\mathrm{ABC}$ triblock copolymers

*Corresponding author. and observed the distorted lamellar [6]. Knoll et al. investigated the thin film of the cylinder-forming triblock copolymers and compared the experimental and theoretical result [7]. Horvat et al. simulated the phase behavior of the cylinder-forming triblock copolymer film [8]. They obtained the morphologies deviating from the bulk and phase diagram of the surface reconstruction by changing the film thickness and surface field. So far, the sphere-forming triblock copolymer film has received much less attention and most of the work in this aspect focused on the packing and packing transition of the spherical domain in the film [9]. A fundamental understanding of morphology reconstruction due to the confinement and the surface field is lacking. In this paper, we employ a mean-field dynamic density functional theory (DDFT) to study confined triblock copolymers with spherical bulk phase. This model has been used extensively in many aspects. Huinink et al. employed this model to study asymmetric diblock copolymers with cylindrical bulk phase confined in the films [3]. Horvat et $a l$. used this model to study the asymmetric triblock copolymer film with cylinder-forming bulk phase [8]. The paper is arranged as follows: in Section 2 we simply introduce the method and parameters. In Section 3, we focus on the morphologies of sphere-forming triblock copolymer films by tuning the surface field and film thickness. Also, the corresponding discussion is given in this section. Finally, the conclusions are presented in Section 4. 


\section{Method}

For our simulation, we used the standard MESODYN code [10]. The MESODYN is based on the mean-field DDFT approach developed by Fraaije et al. [11-16]. In MESODYN, the polymer melt is modeled as a compressible system, consisting of Gaussian chain molecules in a mean-field environment. In this paper, we consider a compressible melt of $n$ ABA triblock copolymers in a volume $V$ at a temperature $T$. The total degree of polymerization of the triblock copolymer is $N$ and the degree of polymerization of block I is $f_{\mathrm{I}} N$, where $f_{\mathrm{I}}$ is the fraction of segments on each chain belonging to type I. Thus, the free energy $F$ used in the MESODYN has the form

$$
F[\rho]=-k T \ln \frac{\varphi^{\mathrm{n}}}{n !}-\sum_{\mathrm{I}} \int_{\mathrm{V}} u_{\mathrm{I}}(\mathbf{r}) \rho_{\mathrm{I}}(\mathbf{r}) \mathrm{d} \mathbf{r}+F^{\mathrm{nid}}[\rho]
$$

where $\phi$ is the single-chain partition function for ideal Gaussian chains in an external field $u_{\mathrm{I}}[12], \rho_{I}$ is the density of the copolymer bead I and nonideal term $F^{\text {nid }}[\rho]$ includes bead-bead and bead-surface interaction as well as Helfand penalty function for compressibility [15]. The details of nonideal term $F^{\text {nid }}[\rho]$ can be found in [3] and [14]. DDFT is used to describe the temporal evolution of the system. Thus, Langevin equation is used to describe the time evolution of the density.

$$
\frac{\partial \rho_{\mathrm{I}}}{\partial t}=M_{\mathrm{I}} \nabla \cdot \rho_{\mathrm{I}} \nabla \mu_{\mathrm{I}}+\eta_{\mathrm{I}}
$$

where $\mu_{I}=\delta F / \delta \rho_{I}$ is intrinsic chemical potential field of a bead of type I, $M_{\mathrm{I}}$ is the mobility parameter of a bead of type I. $\eta_{I}$ is Gaussian noise which satisfies the fluctuation-dissipation theorem[16]. In the neighborhood of hard objects, rigid wall boundary conditions are used. These conditions are implemented by using $\boldsymbol{n} \nabla \mu_{I}=0$, where $\boldsymbol{n}$ is the normal point toward the wall. The same boundary conditions are used for the noise $\eta_{I}$. Utilizing Equation (2) and boundary conditions, these fluxes will drive the system to a steady state, which corresponds to the minima of free energy.

In the paper, we model the polymer film as a collection of Gaussian chain $\mathrm{A}_{8} \mathrm{~B}_{4} \mathrm{~A}_{8}$ with a total length $N=20$. The simulations are completed on a cubic grid of dimensions $X \times Y \times Z=32 \times 32 \times H+1$ with a mesh size $h(h$ $=1 \mathrm{~nm}$ ). One surface is positioned at $z=0$ and the other surface is positioned at $H+1$ because of periodicity of boundary condition. Thus, film thickness is $H$. The interaction between different blocks is characterized by the interaction parameter $\varepsilon_{A B}$ in units of $\mathrm{kJ} / \mathrm{mol}$ and the interaction between blocks and interfaces is characterized by corresponding interaction parameters $\varepsilon_{A M}$ and $\varepsilon_{B M}$. An effective interface-copolymer interaction parameter is equal to $\varepsilon_{M}=\left(\varepsilon_{B M}-\varepsilon_{A M}\right)$, which characterizes the strength of the surface field. In our case, $T=413 \mathrm{~K}$, and $\varepsilon_{B M}=0 \mathrm{~kJ} / \mathrm{mol}$. Thus, surface field strength
$\varepsilon_{M}=-\varepsilon_{A M}$. The mobility parameters were assumed to be equal $\left(M_{\mathrm{A}}=M_{\mathrm{B}}=M\right)$. The dimensionless parameters in MESODYN program are chosen as: the grid parameter $d=a / h=1.1543$ with $a$ the Gaussian bond length, which is optimal ratio for DDFT approach [17]; the time step $\tau=M \Delta t /\left(h^{2} k T\right)=0.5$, which is optimal value [3]; the noise scaling parameter $\Omega=h^{3} / v=100$ with $v$ the bead volume, which is the best numerical performance for pure diffusive system [12,17]; and compressibility parameter $\kappa_{H} / T=8$, which allows small fluctuation. Thus, the phase behavior of the ABA triblock copolymer film is determined by A-B bead interaction parameter $\varepsilon_{A B}$, surface field strength $\varepsilon_{M}$ and the film thickness $H$. In this paper, the unit of $\varepsilon$ is $\mathrm{kJ} / \mathrm{mol}$ and we neglect it from now on. As in [3] and [8], we follow the temporal evolution in the system and stop it when both the free energy and the order parameter do not change significantly. The average simulation time is 5000 dimensionless time steps and the stability of some nonperfect structures is checked by continuing simulations till 20,000 or more time steps.

\section{Results and Discussion}

In order to guarantee that the system is sphere-forming phase in the bulk, the simulation is done with MESODYN in a cubic box with $32 \times 32 \times 32$ grids and periodic boundary condition. We find that B-rich domains of spherical shape form in an A-rich matrix in the range 7.4 $\leq \varepsilon_{A B} \leq 8.4$ in the bulk. Figure 1 shows the isodensity profile of the B-beads for $\varepsilon_{A B}=8$, which is fixed throughout this paper for discussion. The averaged distance between subsequent layers of spheres along $z$ axis is estimated as $c_{0}=6-7$ grid units by MESODYN.

Now, triblock copolymers with spherical phase are put into the thin film. As mentioned above, the phase behavior of the confined ABA triblock copolymer film is controlled by two parameter $\varepsilon_{M}$ and $H$. Considering the effect of the surface field, we fix film thickness with $H=$ 12 , which is compatible with natural bulk domain space $\left(c_{0}\right)$.

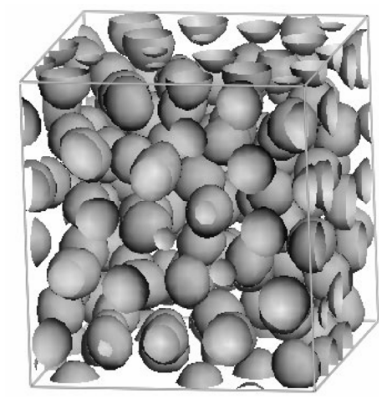

Figure 1. Bulk isodensity profile $\left(\rho_{B} v=0.2\right)$ for $A_{8} B_{4} A_{8}$ triblock copolymer melts at $\varepsilon_{\mathrm{AB}}=8 \mathrm{~kJ} / \mathrm{mol}$. In the following figures, we use the same unit for $\varepsilon$ and will neglect it. 
By varying the surface field strength from 4 to 25 , we obtain the surface structures deviating from the bulk structure, as shown in Figure 2. For $\varepsilon_{\mathrm{M}} \approx 4$, component $\mathrm{B}$ is weakly attracted to the surface and spheres touching the surface are formed (see Figures $\mathbf{2}$ and $\mathbf{3}$ ).

Huinink et al. [3] and Wang et al. [4] have found that neutral wall has weak preference for the short block because of purely entropic effect. We think that the surface can be regarded as neutral in the vicinity of $\varepsilon_{\mathrm{M}}=4$, because the surface attracts B component weakly in the vicinity of $\varepsilon_{\mathrm{M}}=4$. The fact that the neutral wall is not at $\varepsilon_{\mathrm{M}}=0$ has also been observed in [3] and [8]. With the increase of the surface field strength, component B is weakly repelled by the surface and then spheres (S) not connected to surface are formed at $\varepsilon_{\mathrm{M}} \approx 5$ (see Figures 2 and 3). Furthermore, the spheres (S) are somewhat compressed at $\varepsilon_{\mathrm{M}}=5$, compared with those at $\varepsilon_{\mathrm{M}}=4$. Besides, the array of spheres is hexagonal at $\varepsilon_{\mathrm{M}} \approx 4$ and $\varepsilon_{\mathrm{M}} \approx 5$. Continuing to increase the surface field strength, we find that cylinders oriented parallel to the surface $\left(\mathrm{C}_{\|}\right)$appear. Cylinders are compressed with the increase of the surface field and then convert to the perforated lamellae (PL) at $\varepsilon_{\mathrm{M}} \approx 20$. Finally, the perforated lamellae (PL) convert to the lamellae (L) under very large surface field. The appearance of these surface reconstructions mentioned in turn is because that surface-field additivity leads to the decrease of the averaged mean curvature.

Considering the effect of the confinement, we choose surface field strength at $\varepsilon_{\mathrm{M}}=4$, at which the surface can be regarded as neutral. By varying the film thickness from 6 to 18, we obtain the confinement-induced structures, as shown in Figure 4. We find the bulk sphereforming phase is kept for some film thicknesses which are approximately compatible with natural bulk domain space such as $H=6,7,11,12,13,14,17$ and 18 . One layer of spheres is formed for $H=6$ and 7, two layers of spheres are formed for $H=11-13$ and three layers of spheres are formed for $H=17$ and 18. When the film thickness is very incompatible with natural bulk domain space such as $H=8,9,10,15$ and 16 , the bulk sphere-forming phase is frustrated. Moreover, cylinders oriented perpendicular to the surface $\left(\mathrm{C}_{\perp}\right)$ and touching the surface are formed. Here, we define the length of perpendicular cylinder is more than or equal to the length in $H=8 \mathrm{~nm}$, which is an artificial definition (we choose length in $H=8$ as criterion of perpendicular cylinder). Comparing Figures 2 and 4, we find that surface field only induces the cylinders oriented parallel to the surface $\left(\mathrm{C}_{\|}\right)$, perforated lamellae (PL) and lamellae (L) in sphere-forming triblock copolymer thin film. Cylinders oriented perpendicular to the surface $\left(\mathrm{C}_{\perp}\right)$ are induced by incommensurability between the thin film thickness and the bulk period of the triblock copolymers.

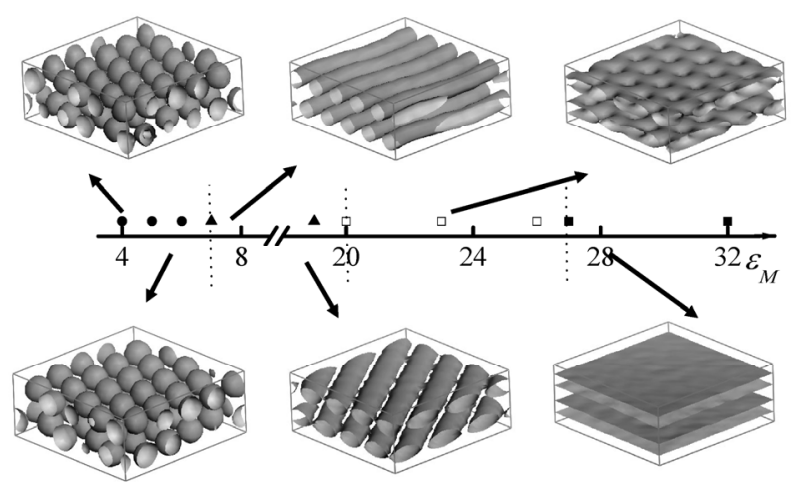

Figure 2. Three-dimensional isodensity profile $\left(\rho_{\mathrm{B}} v=0.2\right)$ for $A_{8} B_{4} A_{8}$ triblock copolymer film with $H=12$ at $\varepsilon_{M}=4,5$, $7,19,20$, and 30 . Solid triangle $(\Delta)$ represents the parallel cylinder; solid ( $\square$ ) and hollow ( $\square$ ) boxes represent the lamella and the perforated lamella respectively, while solid sphere $(\bullet)$ represents the spherical phase.
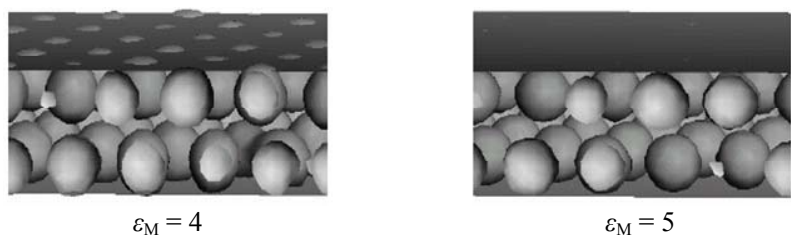

Figure 3. Isodensity profile of the component $B$ where the mask is added at $\varepsilon_{M}=4$ and 5 as a side view of those in Figure 2.

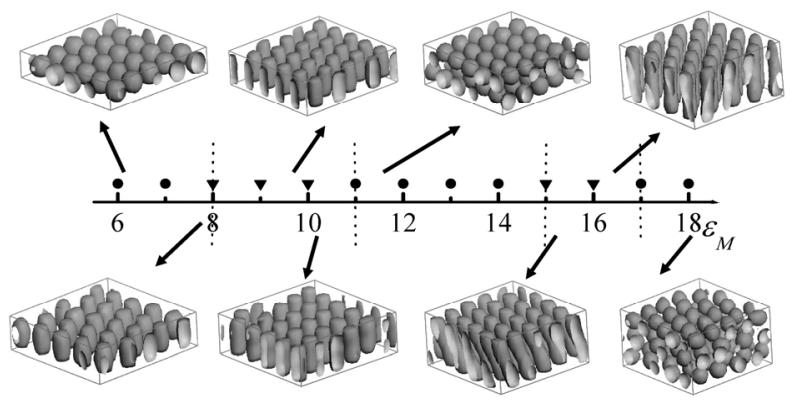

Figure 4. Three-dimensional isodensity profile $\left(\rho_{B} v=0.2\right)$ for $A_{8} B_{4} A_{8}$ triblock copolymer film at $\varepsilon_{M}=4$ with $H=6,8$, $9,10,11,15,16$ and 18 . The solid inverse triangle $(\nabla)$ represents the perpendicular cylinder. The definitions of other symbols are the same as those in Figure 2.

In order to study the cooperating effect of the confinement and the surface field on structures, we simulate the phase behavior of triblock copolymers at different surface fields $\left(4 \leq \varepsilon_{\mathrm{M}} \leq 40\right)$ under different film thickness $(6 \leq H \leq 14)$ and draw the phase diagram of surface reconstruction as shown in Figure 5.

From the phase diagram, we can obtain more information on surface reconstructions. First, spheres (S) do not appear in case of $H=8$ and 9 at all the surface fields. Furthermore, the range of perpendicular cylinders $\left(\mathrm{C}_{\perp}\right)$ exist is wider for $H=8$ and 9 than that for other film 


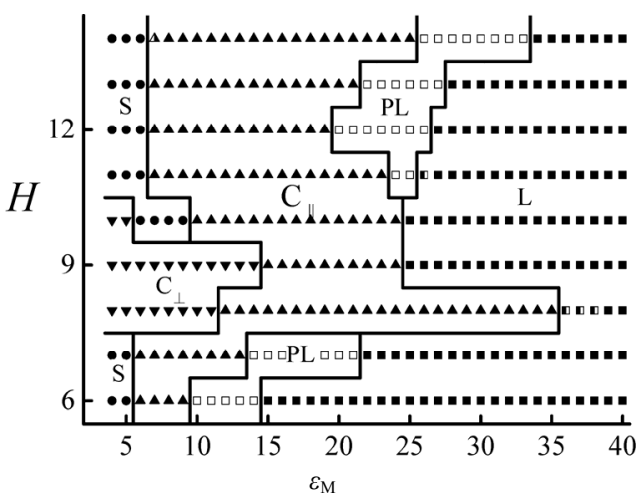

Figure 5. Phase diagram of surface reconstructions, i.e. film thickness $H$ versus surface field strength $\varepsilon_{M}$. The half hollow box indicates that perforated lamella and lamella coexist in the surface layer. The half hollow triangle indicates that spheres and parallel cylinders coexist in the surface layer. The definitions of other symbols are the same as those in Figure 2 and Figure 4.

thicknesses. These phenomena might relate to the incommensurability of sphere layer period of $c_{0}$ with the film thickness. Second, perforated lamellae (PL) do not appear in thin film not only at $H=9$ but also at $H=10$. As a result, the pathway from parallel cylinders to lamellae at $H=9$ and $H=10$ is different from that at other film thicknesses. The intermediate state from parallel cylinders to lamellae is undulated lamellae (connected parallel cylinders) for $H=9$ and 10, while the intermediate stable state is perforated lamellae (PL) for other film thicknesses. Third, the critical surface field required to induce a surface reconstruction with nonspherical phase $\left(\mathrm{C}_{\|}, \mathrm{PL}\right.$ and $\left.\mathrm{L}\right)$ increases with the film thickness which are the integer multiple of a nature layer thickness by comparing $H=6\left(c_{0}\right)$ and $H=12\left(2 c_{0}\right)$. Lamellae (L), perforated lamellae (PL) and parallel cylinders $\left(\mathrm{C}_{\|}\right)$are observed at $\varepsilon_{\mathrm{M}} \approx 15, \varepsilon_{\mathrm{M}} \approx 10$ and $\varepsilon_{\mathrm{M}} \approx 6$, respectively, for the thickness of $H=6$, while the phases are observed correspondingly at $\varepsilon_{\mathrm{M}} \approx 27, \varepsilon_{\mathrm{M}} \approx 20$ and $\varepsilon_{\mathrm{M}} \approx 7$ for the thickness of $H=12$. It indicates that the strength of surface field needed to form these microstructures in thin film is smaller than that in thick film because of the interference effect of two surface field strengths which is in agreement with the conclusion obtained by DDFT in thin film of cylindrical phase [8]. Fourth, the surface fields $\varepsilon_{\mathrm{M}}$, where the lamella (L) is formed at $H=8$, is stronger than that for other thicker films. In general, the strength of surface field needed to form lamellae in thin film is smaller than that in the thick film. However, we find that one lamella is formed for $H=8$ and the width of the lamella is very wider for $H=8$ than for other thicker films, which leads to the chain stretching and the decrease of conformation entropy for $H=8$. Thus, the surface field needs to be strong enough to compensate for unfavored entropic decrease. Finally, there are some coexistence of two phases, for example, $\mathrm{L}$ and $\mathrm{PL}$, and $\mathrm{C}_{\|}$and $\mathrm{S}$ as well. By coexistence of two phases, we can learn how structure evolves from one phase to another phase.

The phase behavior of sphere-forming tirblock copolymer slit is analogous to that of cylinder-forming triblock copolymer slit studied by Horvat et al. [8]. First, the surface reconstructions in sphere-forming triblock copolymer slit are found in cylinder-forming triblock copolymer slit except for spherical phase. It has been proved theoretically that sphere-to-cylinder transition will occur under an external field (confinement, shear, electric field) and hence the transitions due to confinement in cylinder-forming systems can be seen as a subset of the transitions observed in sphere-forming systems. Second, Cylinders oriented perpendicular to the surface $\left(\mathrm{C}_{\perp}\right)$ are observed at the certain thickness which is incompatible with natural bulk domain space for both systems. However, there is an important difference in both systems. For cylinder-forming systems in slit [8], the stable surface reconstructions like $\mathrm{C}_{\|}, \mathrm{PL}$ and $\mathrm{L}$ are found to be nested into each other to respond to the incommensurability. Apparently, the situation for spheres is different. The difference between sphere-forming and cylinder-forming systems may be due to the enhanced flexibility of the sphere-forming system to respond to incommensurability between the film thickness and the domain distance.

\section{Conclusion}

In summary, the effects of the film thickness and the surface field strength on the phase behavior of the sphere-forming copolymer film are investigated. The surface field can induce spheres with hexagonal packing, parallel cylinders, perforated lamellae and lamellae near the surfaces. Cylinders oriented perpendicular to the surface is induced by incommensurability between the thin film thickness and the bulk period of the triblock copolymers. When incommensurability cooperates with the surface field, the phase behavior of sphere-forming triblock copolymer film becomes more complex. Phase diagram of surface reconstruction is constructed and various morphologies are predicted. Comparing the present phase diagram with other relevant phase diagram for the cylinder-forming triblock copolymer film, we find that sphere-forming system in a slit is more flexible to respond to the incommensurability.

\section{Acknowledgements}

We appreciate the financial support from the Fundamental Research Funds for the Central Universities (ZXH 2009D010) and Scientific Research Foundation of Civil Aviation University of China (No. 05qd05s). 


\section{REFERENCES}

[1] Y. Liu, W. Zhao, X. Zheng, A. King, A. Singh, M. H. Rafailovich, J. Sokolov, K. H. Dai, E. J. S. Kramer, A. Schwarz, O. Gebizlioglu and S. K. Sinha, "Surface-Induced Ordering in a Asymmetric Block Copolymers," Macromolecules, Vol. 27, No. 14, 1994, pp. 4000-4010. doi:10.1021/ma00092a047

[2] M. A. van Dijk and R. van den Berg, "Ordering Phenomena in Thin Block Copolymer Films Studied Using Atomic Force Microscopy," Macromolecules, Vol. 28, No. 20, 1995, pp. 6773-6778. doi:10.1021/ma00124a011

[3] H. P. Huinink, J. C. M. Brokken-Zijp, M. A. van Dijk and G. J. A. Sevink, "Surface-Induced Transition in Thin Films of Asymmetric Diblock Copolymers," Macromolecules, Vol. 34, No. 15, 2001, pp. 5325-5330. doi:10.1021/ma000015h

[4] Q. Wang, P. F. Nealey and J. J. de Pablo, "Monte Carlo Simulations of Asymmetric Diblock Copolymer Thin Films Confined between Two Homogeneous Surfaces," Macromolecules, Vol. 34, No. 10, 2001, pp. 3458-3470. doi:10.1021/ma0018751

[5] W. Stocker, J. Beckmann, R. Stadler and J. P. Rabe, "Surface Reconstruction of the Lamellar Morphology in a Symmetric Poly (Styrene-Block-Butadiene-Block-Methyl Methacrylate) Triblock Copolymer: A Tapping Mode Scanning Force Microscope Study," Macromolecules, Vol. 29, No. 23, 1996, pp. 7502-7505. doi: $10.1021 / \mathrm{ma9604000}$

[6] N. Rehse, A. Knoll, R. Magerle and G. Krausch, "Surface Reconstructions of Lamellar ABC Triblock Copolymer Mesostructures," Macromolecules, Vol. 36, No. 9, 2003, pp. 3261-3271. doi:10.1021/ma021134v

[7] A. Knoll, A. Horvat, K. S. Lyakhova, G. Krausch, G. J. A. Sevink, A. V. Zvelindovsky and R. Magerle, "Phase Behavior in Thin Films of Cylinder-Forming Block Copolymers" Physical Review Letters, Vol. 89, No. 3, 2002, pp. 035501/1-4. doi:10.1103/PhysRevLett.89.035501

[8] A. Horvat, K. S. Lyakhova, G. J. A. Sevink, A. V. Zvelindovsky and R. Magerle, "Phase Behavior in Thin Films of Cylinder-forming ABA Block Copolymers: Mesoscale Modelling," Journal of Chemical Physics, Vol. 120, No. 2, 2004, pp. 1117-1126. doi:10.1063/1.1627325

[9] C. B. Tang, J. Bang, G. E. Stein, G. H. Fredrickson, C. J. Hawker, E. J. Kramer, M. Sprung and J. Wang, "Square
Packing and Structural Arrangement of ABC Triblock Copolymer Spheres in Thin Films," Macromolecules, Vol. 41, No 12, 2008, pp. 4328-4339. doi:10.1021/ma800207n

[10] Available from Accelrys Inc., San Diego.

[11] J. G. E. M. Fraaije, "Dynamic Density Functional Theory for Microphase Separation Kinetics of Block Copolymer Melts," Journal of Chemical Physics, Vol. 99, No. 11, 1993, pp. 9202-9212. doi:10.1063/1.465536

[12] J. G. E. M. Fraaije, B. A. C. van Vlimmeren, N. M. Maurits, M. Postma, O. A. Evers, C. Hoffmann, P. Altevogt and G. Goldbeck-Wood, "The Dynamic MeanField Density Functional Method and Its Application to the Mesoscopic Dynamics of Quenched Block Copolymer Melts," Journal of Chemical Physics, Vol. 106, No. 10, 1997, pp. 4260-4269. doi:10.1063/1.473129

[13] B. A. C. van Vlimmeren, N. M. Maurits, A. V. Zvelindovsky, G. J. A. Sevink and J. G. E. M. Fraaije, "Simulation of 3D Mesoscale Structure Formation in Concentrated Aqueous Solution of the Triblock Polymer Surfactants (Ethylene Oxide) $)_{13}$ (Propylene Oxide) $)_{30}$ (Ethylene Oxide) $)_{13}$ and (Propylene Oxide) ${ }_{19}$ (Ethylene Oxide) ${ }_{33}$ (Propylene Oxide) ${ }_{19}$. Application of Dynamic Mean-Field Density Functional Theory," Macromolecules, Vol. 32, No. 3, 1999, pp. 646-656. doi:10.1021/ma980947+

[14] G. J. A. Sevink, A. V. Zvelindovsky, B. A. C. van Vlimmeren, N. M. Maurits and J. G. E. M. Fraaije, "Dynamic of Surface Directed Mesophase Formation in Block Copolymer Melts," Journal of Chemical Physics, Vol. 110, No. 4, 1999, pp. 2250-2256. doi:10.1063/1.477837

[15] N. M. Maurits, B. A. C. van Vlimmeren and J. G. E. M. Fraaije, "Mesoscopic Phase Separation Dynamics of Compressible Copolymer Melts," Physical Review E, Vol. 56, No. 1, 1997, pp. 816-825. doi:10.1103/PhysRevE.56.816

[16] B. A. C. van Vlimmeren and J. G. E. M. Fraaije, "Calculation of Noise Distribution in Mesoscopic Dynamics Models for Phase Separation of Multicomponent Complex Fluids," Computer Physics Communications, Vol. 99, No. 1, 1996, pp. 21-28. doi:10.1016/S0010-4655(96)00125-7

[17] N. M. Maurits, P. Altevogt and O. A. Evers, "Simple Numerical Quadrature Rules for Gaussian Chain Polymer Density Functional Calculations in 3D and Implementation Platforms," Computational and Theoretical Polymer Science, Vol. 6, No. 1-2, 1996, pp. 1-8. 